\title{
Fazit: Verkehrswissenschaft als Gesellschaftswissenschaft
}

\section{Oliver Schwedes}

Der verkehrspolitische Nachhaltigkeitsdiskurs ist geprägt von Hoffnungsträgern. Hoffnungsträger sind die säkularisierte Form des religiösen Heilsbringers, des von Gott gesandten Messias, von dem wir die Erlösung auf Erden erwarten. In den letzten Jahrzehnten gab es eine ganze Reihe solcher Hoffnungsträger, die damit angepriesen wurden, alle Verkehrsprobleme mit einem Schlag zu lösen. Das Carsharing etwa verspricht seit dreißig Jahren durch die kollektive Nutzung eines Autobausteins den privaten Pkw abzulösen und eine nachhaltige Verkehrsentwicklung zu unterstützen, fristet aber bis heute ein Nischendasein und wird auch optimistischsten Prognosen zufolge auf absehbare Zeit keinen signifikanten Beitrag zu einer nachhaltigen Verkehrsentwicklung leisten (ifmo 2016). Es folgte in den 1990er Jahren die erste Welle der Elektromobilität, die nach wenigen Jahren ebenso schnell wieder verschwand wie sie gekommen war, bevor das Elektroauto 2009 wiederentdeckt wurde und ein zweiter e-mobilityHype einsetzte (Schwedes 2020). Zehn Jahre später stehen wir noch immer am Anfang einer Entwicklung, von der wir schon jetzt wissen, dass sie keinen Beitrag zu einer nachhaltigen Verkehrsentwicklung leisten wird, wenn zukünftig alle mit einem privaten Elektroauto fahren werden, anstatt auf den privaten Pkw zu verzichten. In den 2000er Jahren erschien dann vielen Beobachtern die nachwachsende Generation junger Menschen als ein Hoffnungsträger, da sie angeblich weder einen Führerschein machen noch Autofahren wollten, was die Zahlen bis heute nicht hergeben. In jüngster Zeit wird die digitale Revolution im Verkehrssektor als Hoffnungsträger einer nachhaltigen Verkehrsentwicklung begrüßt

O. Schwedes $(\bowtie)$

Technische Universität Berlin, Berlin, Deutschland

E-Mail: oliver.schwedes@tu-berlin.de

(C) Der/die Autor(en) 2021 
und jeder der Einwände etwa gegen das autonome Fahren formuliert als Ketzer geziehen. Der neueste Heilsbringer jedoch ist ein Produkt der sogenannten Plattformökonomie und nennt sich ,Mobility as a Service'. Seine Vertreter*innen versprechen den Kundinnen und Kunden mit ihren privaten Business Modellen jedes gewünschte Mobilitätsangebot aus einer Hand und natürlich wieder nachhaltig (Docherty et al. 2018).

Heilsbringer zeichnen sich durch einen ambivalenten Charakter aus, einerseits verfügen sie zweifellos über ein reales Potential, die mit ihnen verbundenen Hoffnungen $\mathrm{zu}$ erfüllen. Darin unterscheidet sich die säkularisierte Form von ihren heiligen Vorgängern, an die man glauben musste. Andererseits verleiten die Hoffnungsträger dazu, dass man sich auf sie verlässt, ohne nach den gesellschaftlichen Voraussetzungen zu fragen, die erfüllt sein müssen, damit sie ihr reales Potential auch entfalten können. In dieser Hinsicht erfüllen sie noch ganz die Funktion der religiösen Heilsbringer, denen seinerzeit eine überirdische Wirkmacht unterstellt wurde. Indem die Erwartung einer nachhaltigen Verkehrsentwicklung auf die Hoffnungsträger übertragen wird, erfüllen sie eine gesellschaftliche Entlastungsfunktion. Die Politik muss keine unangenehmen Entscheidungen treffen, die Bürger*innen müssen nicht ihren eigenen Lebensstil hinterfragen, der Hoffnungsträger wird es schon richten. Stattdessen wurde die Erwartung einer nachhaltigen Verkehrsentwicklung in den letzten Jahrzehnten immer wieder enttäuscht, ohne das ernsthaft die Frage gestellt wurde, woran das liegt, lieber wenden sich alle Beteiligten dem nächsten Hoffnungsträger zu, von dem sie erwarten, dass er ihre Hoffnungen jetzt aber wirklich erfüllt.

Diese gesellschaftliche Konstellation haben Blühdorn et al. (2020) treffend als nachhaltige Nicht-Nachhaltigkeit bezeichnet und eindrücklich nachgezeichnet, warum die ökologische Transformation der Gesellschaft nicht stattfindet. Dabei haben sie sich von den großen Nachhaltigkeitserzählungen bewusst kritisch distanziert, die von der etablierten Wissenschaft allzu häufig zum Ausgangspunkt ihrer Überlegung gemacht wird, um an die entsprechenden Fördertöpfe zu gelangen.

„Dieser Abstand ist nicht zuletzt deshalb dringend geboten, weil der Verdacht besteht, dass deren soziologisch oft wenig plausiblen Aktivierungs- und Hoffnungserzählungen - ungewollt und unbewusst - vielleicht selbst zur Stabilisierung genau dessen beitragen, wogegen sie sich wenden. Denn entgegen solchen Mobilisierungsnarrativen sind moderne Konsum- und Digitalisierungsgesellschaften tatsächlich nicht bereits auf dem richtigen Weg! Die Wende zur Nachhaltigkeit hat nicht bereits begonnen! Nichts deutet darauf hin, dass die Logik der Nicht-Nachhaltigkeit wirklich ernsthaft angeschlagen wäre. Eine Gesellschaft der Nachhaltigkeit ist - trotz neuer Klimabewegung, Grüner Wahlerfolge und 
vielfältiger ökologischer Modernisierungsprojekte - nirgends in Sicht. Und jede Behauptung, dass es doch so sei, spinnt gewissermaßen das längst abgespielte Stück fort und trägt zur Rechtfertigung des Weiter-So bei“ (ebd., S. 20 f.).

Für die Verkehrswissenschaft folgt daraus, die Rolle der Hoffnungsträger einer nachhaltigen Verkehrsentwicklung kritisch im gesellschaftlichen Kontext zu betrachten und auf jene notwendigen Voraussetzungen hin zu befragen, die erfüllt sein müssen, damit sie die mit ihnen verbundenen Erwartungen erfüllen können. Dann zeigt sich beispielsweise, dass das Autoteilen (Carsharing) darauf angewiesen ist, die Nutzung des privaten Pkw einzuschränken. Stattdessen verteilen die deutschen Automobilkonzerne ihre neusten Modelle als Carsharing-Fahrzeuge getarnt werbewirksam und flächendeckend in den deutschen Innenstädten, dort also, wo sie am wenigsten benötigt werden, weil der öffentliche Verkehr gut ausgebaut ist. Im Ergebnis wird damit die Attraktivität der Autonutzung erhöht, ohne das ein privater Pkw deshalb weichen muss. Nachdem die deutschen Automobilkonzerne die Elektrifizierung jahrzehntelang erfolgreich bekämpft haben, werden die Elektroantriebe jetzt in Verbrennungsfahrzeuge eingebaut, die immer größer und schwerer geworden sind und Batterien erfordern, die zunächst zwei Tonnen in Bewegung setzen müssen, bevor die Reise losgehen kann. In dem Maße wie das private Elektroauto dem alten Leitbild der ,Rennreiselimousine" folgt, die vollbesetzt und ohne Stopp fünfhundert Kilometer mit einer Durchschnittsgeschwindigkeit von einhundert Stundenkilometern überwindet, trägt es nicht zu einer nachhaltigen Verkehrsentwicklung bei. Auch die neue Generation junger Menschen wird nicht nachhaltig geboren, sondern in eine Autogesellschaft. Selbst die Aktivist*innen von Fridays for Future sind angewiesen auf alternative Mobilitätskonzepte, die ihnen ein gutes Leben ermöglichen und falls das Autoteilen dabei eine Rolle spielen wird, werden auch sie gezwungen einen Führerschein machen. Sollten sie zukünftig ein autonomes Fahrzeug nutzen, werden sie unter den gegebenen gesellschaftlichen Rahmenbedingungen mehr Verkehr erzeugen als zuvor, weil die Attraktivität steigt, weitere Strecken mit dem privaten Pkw zurückzulegen, während man zugleich anderen Tätigkeiten nachgeht (Agora Verkehrswende 2020). Schließlich sind die jungen technikaffinen Menschen schon heute die besten Kund*innen jener StartUps, die neue Mobilitätsdienstleistungen wie e-Scooter, e-Kickroller oder e-Bikes anbieten. Ihr legitimes Partikularinteresse besteht darin, die Menschen anzuregen, ihre Fahrzeuge möglichst oft zu nutzen und dabei möglichst viele Kilometer zurückzulegen, was jedoch dem Gemeinwohlinteresse einer nachhaltigen Verkehrsentwicklung fundamental widerspricht. 
Spätestens an dieser Stelle begegnet der kritischen Verkehrswissenschaft der Vorwurf des Defätismus: Könnt ihr auch sagen, wie eine nachhaltige Verkehrsentwicklung zu erreichen ist? Die Beantwortung dieser Frage setzt ein Vorgehen voraus, das sich nicht an einzelnen Hoffnungsträgern abarbeitet, sondern von gesellschaftlichen Problemlagen ausgeht und sich an gemeinsam formulierten Zielen orientiert. Die genannten Hoffnungsträger müssen sich diesen Zielen unterordnen und in eine gesellschaftliche Gesamtstrategie einfügen (Schwedes und Rammert 2020). Unter der Voraussetzung, dass wir im weltweiten Ressourcenverbrauch, der damit einhergehenden Umweltzerstörung sowie dem globalen Klimawandel ein Problem erkennen und eine nachhaltige Verkehrsentwicklung als eine wesentliche Maßnahme begreifen dem zu begegnen, hat das für die genannten Hoffnungsträger ganz konkrete Folgen. Das Carsharing, die kollektive Nutzung des Autobausteins, leistet nur dann einen Beitrag zu einer nachhaltigen Verkehrsentwicklung, wenn zugleich der Besitz des privaten Pkw weitgehend aufgegeben wird. Die elektrisch betriebenen Fahrzeuge müssen so dimensioniert sein und auf eine Art eingesetzt werden, dass sie effektiv genutzt werden können. Das heißt, ihre Höchstgeschwindigkeit müsste deutlich gedrosselt werden, dann könnten sie auch unter Sicherheitsgesichtspunkten kleiner und leichter sein, schließlich sollte ein hoher Besetzungsgrad gewährleistet werden. Die neue Generation junger Menschen muss darin unterstützt werden, dass sie ihr Leben nachhaltig organisieren kann und dabei nicht mehr auf einen privaten Pkw angewiesen ist. Dabei könnten autonome Fahrzeuge im ländlichen Raum eine wichtige Rolle spielen, deren Algorithmen eine permanente Verfügbarkeit unabhängig von privaten Pkw gewährleisten und die Etablierung neuer Mobilitätsroutinen ermöglichen, die eine nachhaltige Verkehrsentwicklung unterstützen. Das ist nur ein Beispiel für eine Vielzahl neuer Mobilitätsdienstleistungen, die im Rahmen einer integrierten verkehrspolitischen Gesamtstrategie gebündelt werden, um im Sinne einer nachhaltigen Verkehrsentwicklung zu weniger Verkehr beizutragen.

Um zu diesen Einsichten vorzudringen, muss Verkehrswissenschaft als Gesellschaftswissenschaft praktiziert werden und sich als kritische Mobilitätswissenschaft etablieren. Eine kritische Mobilitätswissenschaft hat die Aufgabe, jene Macht- und Herrschaftsverhältnisse zu analysieren, die einer nachhaltigen Verkehrsentwicklung entgegenstehen und sie öffentlich zur Diskussion zu stellen (FES 2019). Dem liegt die Vorstellung zugrunde, dass Wissenschaft die Aufgabe hat, die Gesellschaft immer wieder kritisch über ihre eigene Unvollkommenheit aufzuklären (Foucault 1992). Erst wenn dies gelingt, ist es der Wissenschaft möglich, angemessene gesellschaftliche Reformvorschläge zu entwickeln, die sich nicht in oberflächlichen Lippenbekenntnissen erschöpfen, sondern über 
die bestehenden gesellschaftlichen Verhältnisse hinausweisen und damit die allenthalben im Munde geführte große Transformation überhaupt erst denkbar erscheinen lässt. In diesem Sinne haben wir in der kritischen Auseinandersetzung mit der aktuell nicht nachhaltigen Situation im Verkehrssektor, die Voraussetzungen für eine menschengerechte Verkehrsplanung identifiziert. Aus dieser wissenschaftlichen Gesellschaftskritik resultierte die Einsicht in die Notwendigkeit einer grundlegenden Reform des Verkehrssystems: Die Verkehrswende.

Eine kritische Mobilitätsforschung, die Verkehrswissenschaft als Gesellschaftswissenschaft praktiziert, begreift die Verkehrswende nicht als technologische Herausforderung, die mit einer neuen Ampelschaltung für Radfahrer und Fußgänger zu erreichen ist, sondern als Baustein eines gesamtgesellschaftlichen Transformationsprozesses, der das Zusammenleben der Menschen berührt und einen neuen Gesellschaftsvertrag erfordert (Pistor 2020). Hier sehen wir mit Blick auf die Entstehungsgeschichte des öffentlichen Verkehrs hilfreiche Anknüpfungspunkte, die aufgenommen werden können, um ihn zu einer zeitgemäßen Öffentlichen Mobilität weiterzuentwickeln. Während der öffentliche Verkehr seinerzeit im Rahmen der Daseinsvorsorge mit seinem Beitrag für die soziale Integration der Gesellschaft staatlich legitimiert wurde, erfordert die Öffentliche Mobilität eine zivilgesellschaftliche Legitimationsgrundlage, der es gelingt, die soziale mit der ökologischen Frage zu verbinden. Dabei wandelt sich die Rolle des Staates, der sich stärker gegenüber der Gesellschaft öffnet, um den wachsenden Ansprüchen aktiver Teilhabe gerecht zu werden. Das bedeutet nicht, wie immer wieder behauptet wird, dass sich der Staat zurückzieht, vielmehr ist seit langem zu beobachten, dass seine Regelungsdichte in vielen gesellschaftlichen Bereichen zunimmt (Bahle 2003; Bonoli und Natali 2012). Auch die Entwicklung einer Öffentlichen Mobilität, die sich stärker als in der Vergangenheit an den Anforderungen und Bedarfen der Menschen orientiert, erfordert neue staatliche Regelungskompetenzen, die es der öffentlichen Hand erlauben, die politischen, rechtlichen und institutionellen Rahmenbedingungen gesellschaftlicher Partizipation zu gewährleisten. Dementsprechend ist die Neubegründung staatlicher Daseinsvorsorge im Sinne einer Öffentlichen Mobilität besser als eine Neuordnung der ,governance of welfare` zu beschreiben (Bode 2005; Hancké 2014).

Als Folge der Neuordnung staatlicher Wohlfahrtsregulierung sehen sich auch staatliche Steuerungsdisziplinen wie die Verkehrsplanung mit neuen Herausforderungen konfrontiert. Damit Politik und Verwaltung den neuen Anforderungen entsprechen können, muss sich auch die Verkehrsplanung gegenüber der Gesellschaft öffnen und die ehemals ,beplante ' Bevölkerung aktiv in die Planungs- und Entscheidungsprozesse einbeziehen. In der Folge kann sich die 
Verkehrspolitik stärker als in der Vergangenheit an den konkreten Anforderungen und Bedarfen der Menschen orientieren. Damit eröffnen sich neue Gestaltungsmöglichkeiten für eine gemeinwohlorientierte Mobilitätspolitik, die nicht auf die Organisation von wachsenden Verkehrsmengen gerichtet ist, sondern das Ziel verfolgt, gesellschaftliche Teilhabe zu gewährleisten.

Das Konzept der Öffentlichen Mobilität könnte den Ausgangspunkt dafür bilden, der sich abzeichnenden großen Transformation im Verkehrssektor einen entsprechenden gesellschaftlichen Rahmen zu geben, innerhalb dessen eine nachhaltige Verkehrsentwicklung politisch gestaltet werden kann. Am Anfang sollte dabei die Einsicht stehen, dass der aktuelle Wandel von Verkehr und Mobilität äußerst konfliktreich verläuft und die politischen Ziele entsprechend umkämpft sind. Die vielfältigen neuen Mobilitätsdienstleistungen entfalten nicht gleichsam naturwüchsig nachhaltige Entwicklungspotentiale, vielmehr sind sie verbunden mit alten und neuen gesellschaftlichen Akteuren, die jeweils ganz unterschiedliche Partikularinteressen verfolgen. Hinzu kommt, dass die bestehenden Machtund Herrschaftsverhältnisse im Politikfeld Verkehr zunehmend infrage gestellt und von Bürger*innen aktiv bekämpft werden. Ob diese dynamische Gemengelage in einer nachhaltigen Verkehrsentwicklungsstrategie mündet, wird davon abhängen, wie die politischen Kämpfe ausgehen. Vor diesem Hintergrund ist es erforderlich, dass Politik wie Wissenschaft politisch Stellung beziehen anstatt sich wie in der Vergangenheit auf vermeintlich neutrale verkehrspolitische Sachentscheidungen zurückzuziehen.

Die Öffentliche Mobilität in einer demokratischen Gesellschaft erfordert eine öffentliche Repräsentation. Sie muss sich im Recht des Staates ebenso wiederfinden wie in staatlicher Planung und Politik. Demgegenüber repräsentiert der demokratische Rechtsstaat - seine Planung wie auch seine Politik - aktuell vor allem die private Auto-Mobilität. Die anhaltende Schwäche des öffentlichen Verkehrs erklärt sich insbesondere durch seine schwache gesellschaftliche Repräsentation. In der Autogesellschaft ist der öffentliche Verkehr rechtlich ein historisches Relikt (Daseinsvorsorge), planerisch wird er als eine Restgröße behandelt (Mindestangebot) und politisch als ein notwendiges Übel wahrgenommen (Subventionsfall). Vor diesem Hintergrund sind wir der Frage nachgegangen, welche gesellschaftlichen Rahmenbedingungen erfüllt sein müssen, um erfolgreich eine Öffentliche Mobilität zu etablieren, mit der die angestrebte Verkehrswende politisch aktiv gestaltet werden kann. Darüber hinaus haben wir konkrete Hinweise gegeben, welche Bausteine eine Öffentliche Mobilität umfassen müsste, um den Ansprüchen eines reformierten öffentlichen Verkehrs gerecht zu werden. Während das weitreichende Konzept der Öffentliche 
Mobilität vor dem Hintergrund der bescheidenen Rolle des öffentlichen Verkehrs heute noch als Utopie erscheinen mag, verweisen die von uns aufgezeigten neuen Instrumente zur Gestaltung eines menschengerechten Verkehrs auf einen gesellschaftlichen Entwicklungspfad, der politisch konkret entschieden werden kann. Dementsprechend begreifen wir die Öffentliche Mobilität als eine konkrete Utopie, deren Erfolg insbesondere davon abhängen wird, ob eine kritische Verkehrs- und Mobilitätsforschung gesellschaftlich nachgefragt wird.

\section{Literatur}

Agora Verkehrswende. 2020. Die Automatisierung des Automobils und ihre Folgen. Chancen und Risiken selbstfahrender Fahrzeuge für nachhaltige Mobilität. https:// www.agora-verkehrswende.de/fileadmin/Projekte/2020/Automatisierung_des_Automobils/Agora_Verkehrswende_Automatisierung_des_Automobils_und_ihre_Folgen. pdf (06.09.2020).

Bahle, Thomas. 2003. The Changing Institutionalization of Social Services in England and Wales, France and Germany. Is the Welfare State on the Retreat? Journal of European Social Policy 13 (1): 5-20.

Blühdorn, Ingolfur, Felix Butzlaff, Michael Deflorian, Daniel Hausknost, Mirijam Mock. 2020. Nachhaltige Nicht-Nachhaltigkeit. Warum die ökologische Transformation der Gesellschaft nicht stattfindet. Bielefeld: transcript.

Bode, Ingo. 2005. Desorganisation mit System. Die Neuorganisation der , governance of welfare ' in Westeuropa. Berliner Journal für Soziologie 15 (2): 219-239.

Bonoli, Giuliano und David Natali, Hrsg. 2012. The Politics of the New Welfare State. Oxford: University Press.

Docherty, Iain, Greg Marsden, Jillian Anable. 2018. The Governance of Smart Mobility. Transportation Research Part A 115: 114-125.

FES - Friedrich-Ebert-Stiftung. 2019. Hürden auf dem Weg zur Zukunftsstadt. Strategien für eine integrierte Stadt- und Verkehrspolitik. WISO Diskurs 12. https://library.fes.de/ pdf-files/wiso/15881.pdf (06.09.2020).

Foucault, Michel. 1992. Was ist Kritik? Berlin: Merve.

Hancké, Bob Hrsg. 2014. Debating Varieties of Capitalism. Oxford: University Press.

Ifmo - Institut für Mobilitätsforschung. 2016. CarSharing 2025 - Nische oder Mainstream? https://www.ifmo.de/publikationen.html?t=150 (06.09.2020).

Pistor, Katharina. 2020. Der Code des Kapitals. Wie das Recht Reichtum und Ungleichheit schafft. Frankfurt M.: Suhrkamp.

Schwedes, Oliver. 2020: Das Elektroauto. Mobilität im Umbruch, 2. Aufl. Wiesbaden: Springer Vieweg.

Schwedes, Oliver und Alexander Rammert. 2020. Mobilitätsmanagement. Ein neues Handlungsfeld Integrierter Verkehrsplanung. Wiesbaden: Springer VS. 
Open Access Dieses Kapitel wird unter der Creative Commons Namensnennung 4.0 International Lizenz (http://creativecommons.org/licenses/by/4.0/deed.de) veröffentlicht, welche die Nutzung, Vervielfältigung, Bearbeitung, Verbreitung und Wiedergabe in jeglichem Medium und Format erlaubt, sofern Sie den/die ursprünglichen Autor(en) und die Quelle ordnungsgemäß nennen, einen Link zur Creative Commons Lizenz beifügen und angeben, ob Änderungen vorgenommen wurden.

Die in diesem Kapitel enthaltenen Bilder und sonstiges Drittmaterial unterliegen ebenfalls der genannten Creative Commons Lizenz, sofern sich aus der Abbildungslegende nichts anderes ergibt. Sofern das betreffende Material nicht unter der genannten Creative Commons Lizenz steht und die betreffende Handlung nicht nach gesetzlichen Vorschriften erlaubt ist, ist für die oben aufgeführten Weiterverwendungen des Materials die Einwilligung des jeweiligen Rechteinhabers einzuholen.

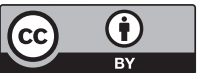

